\title{
Minimum number of additive tuples in groups of prime order
}

\author{
Ostap Chervak Oleg Pikhurko* \\ Mathematics Institute and DIMAP \\ University of Warwick \\ Coventry, U.K. \\ oschervak@gmail.com, o.pikhurko@warwick.ac.uk
}

\author{
Katherine Staden ${ }^{\dagger}$ \\ Mathematical Institute \\ University of Oxford \\ Oxford, U.K. \\ staden@maths.ox.ac.uk
}

Submitted: Oct 5, 2017; Accepted: Jan 30, 2019; Published: Feb 22, 2019

(C) The authors. Released under the CC BY-ND license (International 4.0).

\begin{abstract}
For a prime number $p$ and a sequence of integers $a_{0}, \ldots, a_{k} \in\{0,1, \ldots, p\}$, let $s\left(a_{0}, \ldots, a_{k}\right)$ be the minimum number of $(k+1)$-tuples $\left(x_{0}, \ldots, x_{k}\right) \in A_{0} \times \cdots \times A_{k}$ with $x_{0}=x_{1}+\cdots+x_{k}$, over subsets $A_{0}, \ldots, A_{k} \subseteq \mathbb{Z}_{p}$ of sizes $a_{0}, \ldots, a_{k}$ respectively. We observe that an elegant argument of Samotij and Sudakov can be extended to show that there exists an extremal configuration with all sets $A_{i}$ being intervals of appropriate length. The same conclusion also holds for the related problem, posed by Bajnok, when $a_{0}=\cdots=a_{k}=: a$ and $A_{0}=\cdots=A_{k}$, provided $k$ is not equal 1 modulo $p$. Finally, by applying basic Fourier analysis, we show for Bajnok's problem that if $p \geqslant 13$ and $a \in\{3, \ldots, p-3\}$ are fixed while $k \equiv 1(\bmod p)$ tends to infinity, then the extremal configuration alternates between at least two affine non-equivalent sets.
\end{abstract}

Mathematics Subject Classifications: 11B30, 05D99

\section{Introduction}

Let $\Gamma$ be a given finite Abelian group, with the group operation written additively.

For $A_{0}, \ldots, A_{k} \subseteq \Gamma$, let $s\left(A_{0}, \ldots, A_{k}\right)$ be the number of $(k+1)$-tuples $\left(x_{0}, \ldots, x_{k}\right) \in$ $A_{0} \times \cdots \times A_{k}$ with $x_{0}=x_{1}+\cdots+x_{k}$. If $A_{0}=\cdots=A_{k}:=A$, then we use the shorthand $s_{k}(A):=S\left(A_{0}, \ldots, A_{k}\right)$. For example, $s_{2}(A)$ is the number of $S c h u r$ triples in $A$, that is, ordered triples $\left(x_{0}, x_{1}, x_{2}\right) \in A^{3}$ with $x_{0}=x_{1}+x_{2}$.

For integers $n \geqslant m \geqslant 0$, let $[m, n]:=\{m, m+1, \ldots, n\}$ and $[n]:=[0, n-1]=$ $\{0, \ldots, n-1\}$. For a sequence $a_{0}, \ldots, a_{k} \in[|\Gamma|+1]=\{0,1, \ldots,|\Gamma|\}$, let $s\left(a_{0}, \ldots, a_{k} ; \Gamma\right)$ be

*Supported by ERC grant 306493 and EPSRC grant EP/K012045/1.

${ }^{\dagger}$ Supported by ERC grant 306493 
the minimum of $s\left(A_{0}, \ldots, A_{k}\right)$ over subsets $A_{0}, \ldots, A_{k} \subseteq \Gamma$ of sizes $a_{0}, \ldots, a_{k}$ respectively. Additionally, for $a \in[0, p]$, let $s_{k}(a ; \Gamma)$ be the minimum of $s_{k}(A)$ over all $a$-sets $A \subseteq \Gamma$.

The question of finding the maximal size of a sum-free subset of $\Gamma$ (i.e. the maximum $a$ such that $s_{2}(a ; \Gamma)=0$ ) originated in a paper of Erdös [2] in 1965 and took 40 years before it was resolved in full generality by Green and Ruzsa [3]. Huczynska, Mullen and Yucas [4], and later Samotij and Sudakov [7], introduced the problem of finding $s_{2}(a ; \Gamma)$. This function has a resemblance to some classical questions in extremal combinatorics, where one has to minimise the number of forbidden configurations, see [7, Section 1] for more details.

Huczynska, Mullen and Yucas [4] were able to solve the $s_{2}$-problem for $\Gamma=\mathbb{Z}_{p}$, where $p$ is prime and $\mathbb{Z}_{p}$ is the cyclic group of order $p$. Samotij and Sudakov [7] solved the $s_{2}$-problem for various groups, including a different proof of the $\mathbb{Z}_{p}$ case. Bajnok $[1$, Problem G.48] suggested the more general problem of considering $s_{k}(a ; \Gamma)$. Since even the $s_{2}$-case is still wide open in full generality, Bajnok [1, Problem G.49] proposed, as a possible first step, to consider $s_{k}\left(a ; \mathbb{Z}_{p}\right)$, where $p$ is prime and $k \geqslant 3$.

This paper concentrates on the latter question of Bajnok. Therefore, let $p$ be a fixed prime and let, by default, the underlying group be $\mathbb{Z}_{p}$, which we identify with the additive group of residues modulo $p$ (also using the multiplicative structure on it when this is useful). In particular, we write $s\left(a_{0}, \ldots, a_{k}\right):=s\left(a_{0}, \ldots, a_{k} ; \mathbb{Z}_{p}\right)$ and $s_{k}(a):=s_{k}\left(a ; \mathbb{Z}_{p}\right)$. Since the case $p=2$ is trivial, let us assume that $p \geqslant 3$. By an $m$-term arithmetic progression (or $m$-AP for short) we mean a set of the form $\{x, x+d, \ldots, x+(m-1) d\}$ for some $x, d \in \mathbb{Z}_{p}$ with $d \neq 0$. We call $d$ the difference. For $I \subseteq \mathbb{Z}_{p}$ and $x, y \in \mathbb{Z}_{p}$, write $x \cdot I+y:=\{x \cdot z+y \mid z \in I\}$.

As we already mentioned, the case $k=2$ has been completely resolved: Huczynska, Mullen and Yucas determined $s_{2}(a)$, and Samotij and Sudakov [7] showed that, when $s_{2}(a)>0$, then the $a$-sets that achieve the minimum are exactly those of the form $\xi \cdot I$ with $\xi \in \mathbb{Z}_{p} \backslash\{0\}$, where $I$ consists of the residues modulo $p$ of $a$ integers closest to $\frac{p-1}{2} \in \mathbb{Z}$. Each such set is an arithmetic progression; its difference can be any non-zero value but the initial element has to be carefully chosen.

Here we propose a generalisation of Bajnok's question, namely to investigate the function $s\left(a_{0}, \ldots, a_{k}\right)$. First, by adopting the elegant argument of Samotij and Sudakov [7], we show that at least one extremal configuration consists of $k+1$ arithmetic progressions with the same difference. Note that since

$$
s\left(A_{0}, \ldots, A_{k}\right)=s\left(\xi \cdot A_{0}+\eta_{0}, \ldots, \xi \cdot A_{k}+\eta_{k}\right), \quad \text { for } \xi \neq 0 \text { and } \eta_{0}=\eta_{1}+\cdots+\eta_{k},
$$

finding such arithmetic progressions reduces to finding progressions with difference 1 (and starting element 0 for some $k$ of the sets).

Theorem 1. For arbitrary $k \geqslant 1$ and $a_{0}, \ldots, a_{k} \in[0, p]$, there is $t \in \mathbb{Z}_{p}$ such that

$$
s\left(a_{0}, \ldots, a_{k}\right)=s\left(\left[a_{0}\right]+t,\left[a_{1}\right], \ldots,\left[a_{k}\right]\right) .
$$

In particular, if $a_{0}=\cdots=a_{k}=: a$, then one extremal configuration consists of $A_{1}=\cdots=A_{k}=[a]$ and $A_{0}=[t, t+a-1]$ for some $t \in \mathbb{Z}_{p}$. Given this, one can write 
down some formulas for $s\left(a_{0}, \ldots, a_{k}\right)$ in terms of $a_{0}, \ldots, a_{k}$ involving summation (based on (3) or a version of (13)) but there does not seem to be a closed form in general.

If $k \not \equiv 1(\bmod p)$, then by taking $\xi:=1, \eta_{1}:=\cdots:=\eta_{k}:=-t(k-1)^{-1}$, and $\eta_{0}:=-k t(k-1)^{-1}$ in $(1)$, we can get another extremal configuration where all sets are the same: $A_{0}+\eta_{0}=\cdots=A_{k}+\eta_{k}$. Thus Theorem 1 directly implies the following corollary.

Corollary 2. For every $k \geqslant 2$ with $k \neq 1(\bmod p)$ and $a \in[0, p]$, there is $t \in \mathbb{Z}_{p}$ such that $s_{k}(a)=s_{k}([t, t+a-1])$.

Unfortunately, if $k \geqslant 3$, then there may be sets $A$ different from APs that attain equality in Corollary 2 with $s_{k}(|A|)>0$ (which is in contrast to the case $k=2$ ). For example, our (non-exhaustive) search showed that this happens already for $p=17$, when

$$
s_{3}(14)=2255=s_{3}([-1,12])=s_{3}([6,18] \cup\{3\}) .
$$

Also, already the case $k=2$ of the more general Theorem 1 exhibits extra solutions. Of course, by analysing the proof of Theorem 1 or Corollary 2 one can write a necessary and sufficient condition for the cases of equality. We do this in Section 2; in some cases this condition can be simplified.

However, by using basic Fourier analysis on $\mathbb{Z}_{p}$, we can describe the extremal sets for Corollary 2 when $k \not \equiv 1(\bmod p)$ is sufficiently large.

Theorem 3. Let a prime $p \geqslant 7$ and an integer $a \in[3, p-3]$ be fixed, and let $k \not \equiv 1$ $(\bmod p)$ be sufficiently large. Then there exists $t \in \mathbb{Z}_{p}$ for which the only $s_{k}(a)$-extremal sets are $\xi \cdot[t, t+a-1]$ for all non-zero $\xi \in \mathbb{Z}_{p}$.

Problem 4. Find a 'good' description of all extremal families for Corollary 2 (or perhaps Theorem 1) for $k \geqslant 3$.

While Corollary 2 provides an example of an $s_{k}(a)$-extremal set for $k \not \equiv 1(\bmod p)$, the case $k \equiv 1(\bmod p)$ of the $s_{k}(a)$-problem turns out to be somewhat special. Here, translating a set $A$ has no effect on the quantity $s_{k}(A)$. More generally, let $\mathcal{A}$ be the group of all invertible affine transformations of $\mathbb{Z}_{p}$, that is, it consists of maps $x \mapsto \xi \cdot x+\eta$, $x \in \mathbb{Z}_{p}$, for $\xi, \eta \in \mathbb{Z}_{p}$ with $\xi \neq 0$. Then

$$
s_{k}(\alpha(A))=s_{k}(A), \quad \text { for every } k \equiv 1(\bmod p) \text { and } \alpha \in \mathcal{A} .
$$

Let us call two subsets $A, B \subseteq \mathbb{Z}_{p}$ (affine) equivalent if there is $\alpha \in \mathcal{A}$ with $\alpha(A)=B$. By (2), we need to consider sets only up to this equivalence. Trivially, any two subsets of $\mathbb{Z}_{p}$ of size $a$ are equivalent if $a \leqslant 2$ or $a \geqslant p-2$.

Again using Fourier analysis on $\mathbb{Z}_{p}$, we show the following result.

Theorem 5. Let a prime $p \geqslant 7$ and an integer $a \in[3, p-3]$ be fixed, and let $k \equiv 1$ $(\bmod p)$ be sufficiently large. Then the following statements hold for the $s_{k}(a)$-problem.

1. If $a$ and $k$ are both even, then $[a]$ is the unique (up to affine equivalence) extremal set. 
2. If at least one of $a$ and $k$ is odd, define $I^{\prime}:=[a-1] \cup\{a\}=\{0, \ldots, a-2, a\}$. Then

(a) $s_{k}(a)<s_{k}([a])$ for all large $k$;

(b) $I^{\prime}$ is the unique extremal set for infinitely many $k$;

(c) $s_{k}(a)<s_{k}\left(I^{\prime}\right)$ for infinitely many $k$, provided there are at least three nonequivalent a-subsets of $\mathbb{Z}_{p}$.

It is not hard to see that there are at least three non-equivalent $a$-subsets of $\mathbb{Z}_{p}$ if and only if $p \geqslant 13$ and $a \in[3, p-3]$, or $p \geqslant 11$ and $a \in[4, p-4]$. Thus Theorem 5 characterises pairs $(p, a)$ for which there exists an $a$-subset $A$ which is $s_{k}(a)$-extremal for all large $k \equiv 1(\bmod p)$.

Corollary 6. Let $p$ be a prime and $a \in[0, p]$. There is an a-subset $A \subseteq \mathbb{Z}_{p}$ with $s_{k}(A)=$ $s_{k}(a)$ for all large $k \equiv 1(\bmod p)$ if and only if $a \leqslant 2$, or $a \geqslant p-2$, or $p \in\{7,11\}$ and $a=3$.

As is often the case in mathematics, a new result leads to further open problems.

Problem 7. Given $a \in[3, p-3]$, find a 'good' description of all $a$-subsets of $\mathbb{Z}_{p}$ that are $s_{k}(a)$-extremal for at least one (resp. infinitely many) values of $k \equiv 1(\bmod p)$.

Problem 8. Is it true that for every $a \in[3, p-3]$ there is $k_{0}$ such that for all $k \geqslant k_{0}$ with $k \equiv 1(\bmod p)$, any two $s_{k}(a)$-extremal sets are affine equivalent?

\section{Proof of Theorem 1}

Here we prove Theorem 1 by adopting the proof of Samotij and Sudakov [7].

Let $A_{1}, \ldots, A_{k}$ be subsets of $\mathbb{Z}_{p}$. Define $\sigma\left(x ; A_{1}, \ldots, A_{k}\right)$ as the number of $k$-tuples $\left(x_{1}, \ldots, x_{k}\right) \in A_{1} \times \cdots \times A_{k}$ with $x=x_{1}+\cdots+x_{k}$. Also, for an integer $r \geqslant 0$, let

$$
\begin{aligned}
N_{r}\left(A_{1}, \ldots, A_{k}\right) & :=\left\{x \in \mathbb{Z}_{p} \mid \sigma\left(x ; A_{1}, \ldots, A_{k}\right) \geqslant r\right\}, \\
n_{r}\left(A_{1}, \ldots, A_{k}\right) & :=\left|N_{r}\left(A_{1}, \ldots, A_{k}\right)\right| .
\end{aligned}
$$

These notions are related to our problem because of the following easy identity:

$$
s\left(A_{0}, \ldots, A_{k}\right)=\sum_{r=1}^{\infty}\left|A_{0} \cap N_{r}\left(A_{1}, \ldots, A_{k}\right)\right| .
$$

Let an interval mean an arithmetic progression with difference 1, i.e. a subset $I$ of $\mathbb{Z}_{p}$ of form $\{x, x+1, \ldots, x+y\}$. Its centre is $x+y / 2 \in \mathbb{Z}_{p}$; it is unique if $I$ is proper (that is, $0<|I|<p)$. Note the following easy properties of the sets $N_{r}$ :

1. These sets are nested:

$$
N_{0}\left(A_{1}, \ldots, A_{k}\right)=\mathbb{Z}_{p} \supseteq N_{1}\left(A_{1}, \ldots, A_{k}\right) \supseteq N_{2}\left(A_{1}, \ldots, A_{k}\right) \supseteq \ldots
$$


2. If each $A_{i}$ is an interval with centre $c_{i}$, then $N_{r}\left(A_{1}, \ldots, A_{k}\right)$ is an interval with centre $c_{1}+\cdots+c_{k}$.

We will also need the following result of Pollard [6, Theorem 1].

Theorem 9. Let $p$ be a prime, $k \geqslant 1$, and $A_{1}, \ldots, A_{k}$ be subsets of $\mathbb{Z}_{p}$ of sizes $a_{1}, \ldots, a_{k}$. Then for every integer $r \geqslant 1$, we have

$$
\sum_{i=1}^{r} n_{i}\left(A_{1}, \ldots, A_{k}\right) \geqslant \sum_{i=1}^{r} n_{i}\left(\left[a_{1}\right], \ldots,\left[a_{k}\right]\right) .
$$

Proof of Theorem 1. Let $A_{0}, \ldots, A_{k}$ be some extremal sets for the $s\left(a_{0}, \ldots, a_{k}\right)$-problem. We can assume that $0<a_{0}<p$, because $s\left(A_{0}, \ldots, A_{k}\right)$ is 0 if $a_{0}=0$ and $\prod_{i=1}^{k} a_{i}$ if $a_{0}=p$, regardless of the choice of the sets $A_{i}$.

Since $n_{0}\left(\left[a_{1}\right], \ldots,\left[a_{k}\right]\right)=p>p-a_{0}$ while $n_{r}\left(\left[a_{1}\right], \ldots,\left[a_{k}\right]\right)=0<p-a_{0}$ when, for example, $r>\prod_{i=1}^{k-1} a_{i}$, there is a (unique) integer $r_{0} \geqslant 0$ such that

$$
\begin{aligned}
& n_{r}\left(\left[a_{1}\right], \ldots,\left[a_{k}\right]\right)>p-a_{0}, \quad \text { all } r \in\left[0, r_{0}\right], \\
& n_{r}\left(\left[a_{1}\right], \ldots,\left[a_{k}\right]\right) \leqslant p-a_{0}, \quad \text { all integers } r \geqslant r_{0}+1 .
\end{aligned}
$$

The nested intervals $N_{1}\left(\left[a_{1}\right], \ldots,\left[a_{k}\right]\right) \supseteq N_{2}\left(\left[a_{1}\right], \ldots,\left[a_{k}\right]\right) \supseteq \ldots$ have the same centre $c:=\left(\left(a_{1}-1\right)+\cdots+\left(a_{k}-1\right)\right) / 2$. Thus there is a translation $I:=\left[a_{0}\right]+t$ of $\left[a_{0}\right]$, with $t$ independent of $r$, which has as small as possible intersection with each $N_{r}$-interval above given their sizes, that is,

$$
\left|I \cap N_{r}\left(\left[a_{1}\right], \ldots,\left[a_{k}\right]\right)\right|=\max \left\{0, n_{r}\left(\left[a_{1}\right], \ldots,\left[a_{k}\right]\right)+a_{0}-p\right\}, \quad \text { for all } r \in \mathbb{N} .
$$

This and Pollard's theorem give the following chain of inequalities:

$$
\begin{aligned}
s\left(A_{0}, \ldots, A_{k}\right) & \stackrel{(3)}{=} \sum_{i=1}^{\infty}\left|A_{0} \cap N_{i}\left(A_{1}, \ldots, A_{k}\right)\right| \\
& \geqslant \sum_{i=1}^{r_{0}}\left|A_{0} \cap N_{i}\left(A_{1}, \ldots, A_{k}\right)\right| \\
& \geqslant \sum_{i=1}^{r_{0}}\left(n_{i}\left(A_{1}, \ldots, A_{k}\right)+a_{0}-p\right) \\
& \geqslant \sum_{i=1}{ }^{\frac{T_{0}}{} 9}\left(n_{i}\left(\left[a_{1}\right], \ldots,\left[a_{k}\right]\right)+a_{0}-p\right) \\
& \stackrel{(5)-(6)}{=} \sum_{i=1}^{\infty} \max \left\{0, n_{i}\left(\left[a_{1}\right], \ldots,\left[a_{k}\right]\right)+a_{0}-p\right\} \\
& \stackrel{(7)}{=} \sum_{i=1}^{\infty}\left|I \cap N_{i}\left(\left[a_{1}\right], \ldots,\left[a_{k}\right]\right)\right| \\
& \stackrel{(3)}{=} \quad s\left(I,\left[a_{1}\right], \ldots,\left[a_{k}\right]\right),
\end{aligned}
$$

giving the required. 
Let us write a necessary and sufficient condition for equality in Theorem 1 in the case $a_{0}, \ldots, a_{k} \in[1, p-1]$. Let $r_{0} \geqslant 0$ be defined by (5)-(6). Then, by (4), a sequence $A_{0}, \ldots, A_{k} \subseteq \mathbb{Z}_{p}$ of sets of sizes respectively $a_{0}, \ldots, a_{k}$ is extremal if and only if

$$
\begin{aligned}
A_{0} \cap N_{r_{0}+1}\left(A_{1}, \ldots, A_{k}\right) & =\varnothing, \\
A_{0} \cup N_{r_{0}}\left(A_{1}, \ldots, A_{k}\right) & =\mathbb{Z}_{p}, \\
\sum_{i=1}^{r_{0}} n_{i}\left(A_{1}, \ldots, A_{k}\right) & =\sum_{i=1}^{r_{0}} n_{i}\left(\left[a_{1}\right], \ldots,\left[a_{k}\right]\right) .
\end{aligned}
$$

Let us now concentrate on the case $k=2$, trying to simplify the above condition. We can assume that no $a_{i}$ is equal to 0 or $p$ (otherwise the choice of the other two sets has no effect on $s\left(A_{0}, A_{1}, A_{2}\right)$ and every triple of sets of sizes $a_{0}, a_{1}$ and $a_{2}$ is extremal). Also, as in [7], let us exclude the case $s\left(a_{0}, a_{1}, a_{2}\right)=0$, as then there are in general many extremal configurations. Note that $s\left(a_{0}, a_{1}, a_{2}\right)=0$ if and only if $r_{0}=0$; also, by the Cauchy-Davenport theorem (the special case $k=2$ and $r=1$ of Theorem 9), this is equivalent to $a_{1}+a_{2}-1 \leqslant p-a_{0}$. Assume by symmetry that $a_{1} \leqslant a_{2}$. Note that (5) implies that $r_{0} \leqslant a_{1}$.

The condition in (10) states that we have equality in Pollard's theorem. A result of Nazarewicz, O'Brien, O'Neill and Staples [5, Theorem 3] characterises when this happens (for $k=2$ ), which in our notation is the following.

Theorem 10. For $k=2$ and $1 \leqslant r_{0} \leqslant a_{1} \leqslant a_{2}<p$, we have equality in (10) if and only if at least one of the following conditions holds:

1. $r_{0}=a_{1}$

2. $a_{1}+a_{2} \geqslant p+r_{0}$,

3. $a_{1}=a_{2}=r_{0}+1$ and $A_{2}=g-A_{1}$ for some $g \in \mathbb{Z}_{p}$,

4. $A_{1}$ and $A_{2}$ are arithmetic progressions with the same difference.

Let us try to write more explicitly each of these four cases, when combined with (8) and (9).

First, consider the case $r_{0}=a_{1}$. We have $N_{a_{1}}\left(\left[a_{1}\right],\left[a_{2}\right]\right)=\left[a_{1}-1, a_{2}-1\right]$ and thus $n_{a_{1}}\left(\left[a_{1}\right],\left[a_{2}\right]\right)=a_{2}-a_{1}+1>p-a_{0}$, that is, $a_{2}-a_{1} \geqslant p-a_{0}$. The condition (8) holds automatically since $N_{i}\left(A_{1}, A_{2}\right)=\varnothing$ whenever $i>\left|A_{1}\right|$. The other condition (9) may be satisfied even when none of the sets $A_{i}$ is an arithmetic progression (for example, take $p=13, A_{1}=\{0,1,3\}, A_{2}=\{0,2,3,5,6,7,9,10\}$ and let $A_{0}$ be the complement of $\left.N_{3}\left(A_{1}, A_{2}\right)=\{3,6,10\}\right)$. We do not see any better characterisation here, apart from stating that (9) holds.

Next, suppose that $a_{1}+a_{2} \geqslant p+r_{0}$. Then, for any two sets $A_{1}$ and $A_{2}$ of sizes $a_{1}$ and $a_{2}$, we have $N_{r_{0}}\left(A_{1}, A_{2}\right)=\mathbb{Z}_{p}$; thus (9) holds automatically. Similarly to the previous case, there does not seem to be a nice characterisation of (8). For example, (8) may hold 
even when none of the sets $A_{i}$ is an AP: e.g. let $p=11, A_{1}=A_{2}=\{0,1,2,3,4,5,7\}$, and let $A_{0}=\{0,2,10\}$ be the complement of $N_{4}\left(A_{1}, A_{2}\right)=\{1,3,4,5,6,7,8,9\}$ (here $r_{0}=3$ ).

Next, suppose that we are in the third case. The primality of $p$ implies that $g \in \mathbb{Z}_{p}$ satisfying $A_{2}=g-A_{1}$ is unique and thus $N_{r_{0}+1}\left(A_{1}, A_{2}\right)=\{g\}$. Therefore (8) is equivalent to $A_{0} \not \supset g$. Also, note that if $I_{1}$ and $I_{2}$ are intervals of size $r_{0}+1$, then $n_{r_{0}}\left(I_{1}, I_{2}\right)=3$. By the definition of $r_{0}$, we have $p-2 \leqslant a_{0} \leqslant p-1$. Thus we can choose any integer $r_{0} \in[1, p-2]$ and $\left(r_{0}+1\right)$-sets $A_{2}=g-A_{1}$, and then let $A_{0}$ be obtained from $\mathbb{Z}_{p}$ by removing $g$ and at most one further element of $N_{r_{0}}\left(A_{1}, A_{2}\right)$. Here, $A_{0}$ is always an AP (as a subset of $\mathbb{Z}_{p}$ of size $a_{0} \geqslant p-2$ ) but $A_{1}$ and $A_{2}$ need not be.

Finally, let us show that if $A_{1}$ and $A_{2}$ are arithmetic progressions with the same difference $d$ and we are not in Case 1 nor 2 of Theorem 10, then $A_{0}$ is also an arithmetic progression whose difference is $d$. By (1), it is enough to prove this when $A_{1}=\left[a_{1}\right]$ and $A_{2}=\left[a_{2}\right]$ (and $d=1$ ). Since $a_{1}+a_{2} \leqslant p-1+r_{0}$ and $r_{0}+1 \leqslant a_{1} \leqslant a_{2}$, we have that

$$
\begin{aligned}
N_{r_{0}}\left(A_{1}, A_{2}\right) & =\left[r_{0}-1, a_{1}+a_{2}-r_{0}-1\right] \\
N_{r_{0}+1}\left(A_{1}, A_{2}\right) & =\left[r_{0}, a_{1}+a_{2}-r_{0}-2\right]
\end{aligned}
$$

have sizes respectively $a_{1}+a_{2}-2 r_{0}+1<p$ and $a_{1}+a_{2}-2 r_{0}-1>0$. We see that $N_{r_{0}+1}\left(A_{1}, A_{2}\right)$ is obtained from the proper interval $N_{r_{0}}\left(A_{1}, A_{2}\right)$ by removing its two endpoints. Thus $A_{0}$, which is sandwiched between the complements of these two intervals by (8)-(9), must be an interval too. (And, conversely, every such triple of intervals is extremal.)

\section{The proof of Theorems 3 and 5}

Let us recall the basic definitions and facts of Fourier analysis on $\mathbb{Z}_{p}$. For a more detailed treatment of this case, see e.g. [8, Chapter 2]. Write $\omega:=e^{2 \pi i / p}$ for the $p^{\text {th }}$ root of unity. Given a function $f: \mathbb{Z}_{p} \rightarrow \mathbb{C}$, we define its Fourier transform to be the function $\widehat{f}: \mathbb{Z}_{p} \rightarrow \mathbb{C}$ given by

$$
\widehat{f}(\gamma):=\sum_{x=0}^{p-1} f(x) \omega^{-x \gamma}, \quad \text { for } \gamma \in \mathbb{Z}_{p} .
$$

Parseval's identity states that

$$
\sum_{x=0}^{p-1} f(x) \overline{g(x)}=\frac{1}{p} \sum_{\gamma=0}^{p-1} \widehat{f}(\gamma) \overline{\hat{g}(\gamma)} .
$$

The convolution of two functions $f, g: \mathbb{Z}_{p} \rightarrow \mathbb{C}$ is given by

$$
(f * g)(x):=\sum_{y=0}^{p-1} f(y) g(x-y) .
$$

It is not hard to show that the Fourier transform of a convolution equals the product of Fourier transforms, i.e.

$$
\widehat{f_{1} * \ldots * f_{k}}=\widehat{f_{1}} \cdot \ldots \cdot \widehat{f_{k}} \text {. }
$$


We write $f^{* k}$ for the convolution of $f$ with itself $k$ times. (So, for example, $f^{* 2}=f * f$.) Denote by $\mathbb{1}_{A}$ the indicator function of $A \subseteq \mathbb{Z}_{p}$ which assumes value 1 on $A$ and 0 on $\mathbb{Z}_{p} \backslash A$. We will call $\widehat{\mathbb{1}}_{A}(0)=|A|$ the trivial Fourier coefficient of $A$. Since the Fourier transform behaves very nicely with respect to convolution, it is not surprising that our parameter of interest, $s_{k}(A)$, can be written as a simple function of the Fourier coefficients of $\mathbb{1}_{A}$. Indeed, let $A \subseteq \mathbb{Z}_{p}$ and $x \in \mathbb{Z}_{p}$. Then the number of tuples $\left(a_{1}, \ldots, a_{k}\right) \in A^{k}$ such that $a_{1}+\ldots+a_{k}=x$ (which is $\sigma(x ; A, \ldots, A)$ in the notation of Section 2) is precisely $\mathbb{1}_{A}^{* k}(x)$. The function $s_{k}(A)$ counts such a tuple if and only if its sum $x$ also lies in $A$. Thus,

$$
s_{k}(A)=\sum_{x=0}^{p-1} \mathbb{1}_{A}^{* k}(x) \mathbb{1}_{A}(x) \stackrel{(11)}{=} \frac{1}{p} \sum_{\gamma=0}^{p-1} \widehat{\mathbb{1}_{A}^{* k}}(\gamma) \overline{\widehat{\mathbb{1}_{A}}(\gamma)} \stackrel{(12)}{=} \frac{1}{p} \sum_{\gamma=0}^{p-1}\left(\widehat{\mathbb{1}_{A}}(\gamma)\right)^{k} \overline{\widehat{\mathbb{1}_{A}}(\gamma)} .
$$

Since every set $A \subseteq \mathbb{Z}_{p}$ of size $a$ has the same trivial Fourier coefficient (namely $\widehat{\mathbb{1}_{A}}(0)=a$ ), let us re-write (13) as

$$
p s_{k}(A)-a^{k+1}=\sum_{\gamma=1}^{p-1}\left(\widehat{\mathbb{1}_{A}}(\gamma)\right)^{k} \overline{\mathbb{1}_{A}(\gamma)}=: F(A)
$$

Thus we need to minimise $F(A)$ (which is a real number for any $A$ ) over $a$-subsets $A \subseteq \mathbb{Z}_{p}$. To do this when $k$ is sufficiently large, we will consider the largest in absolute value nontrivial Fourier coefficient $\widehat{\mathbb{1}_{A}}(\gamma)$ of an $a$-subset $A$. Indeed, the term $\left(\widehat{\mathbb{1}_{A}}(\gamma)\right)^{k} \widehat{\widehat{\mathbb{1}_{A}}(\gamma)}$ will dominate $F(A)$, so if it has strictly negative real part, then $F(A)<F(B)$ for all $a$ subsets $B \subseteq \mathbb{Z}_{p}$ with $\max _{\delta \neq 0}\left|\widehat{\mathbb{1}_{B}}(\delta)\right|<\left|\widehat{\mathbb{1}_{A}}(\gamma)\right|$.

Given $a \in[p-1]$, let

$$
I:=[a]=\{0, \ldots, a-1\} \quad \text { and } \quad I^{\prime}:=[a-1] \cup\{a\}=\{a, \ldots, a-2, a\} .
$$

In order to prove Theorems 3 and 5, we will make some preliminary observations about these special sets. The set of $a$-subsets which are affine equivalent to $I$ is precisely the set of $a$-APs.

Next we will show that

$$
F(I)=2 \sum_{\gamma=1}^{(p-1) / 2}(-1)^{\gamma(a-1)(k-1)}\left|\widehat{\mathbb{1}}_{I}(\gamma)\right|^{k+1} \quad \text { if } k \equiv 1 \quad(\bmod p) .
$$

Note that $(-1)^{\gamma(a-1)(k-1)}$ equals $(-1)^{\gamma}$ if both $a, k$ are even and 1 otherwise. To see (15), let $\gamma \in\left\{1, \ldots, \frac{p-1}{2}\right\}$ and write $\widehat{\mathbb{1}_{I}}(\gamma)=r e^{\theta i}$ for some $r>0$ and $0 \leqslant \theta<2 \pi$. Then $\theta$ is the midpoint of $0,-2 \pi \gamma / p, \ldots,-2(a-1) \gamma \pi / p$, i.e. $\theta=-\pi(a-1) \gamma / p$. Choose $s \in \mathbb{N}$ such that $k=s p+1$. Then

$$
\left(\widehat{\mathbb{1}_{I}}(\gamma)\right)^{k} \overline{\mathbb{1}_{I}(\gamma)}=\left(r e^{-\pi i(a-1) \gamma / p}\right)^{k} r e^{\pi i(a-1) \gamma / p}=r^{k+1} e^{-\pi i(a-1) \gamma s},
$$


and $e^{-\pi i(a-1) s}$ equals 1 if $(a-1) s$ is even, and -1 if $(a-1) s$ is odd. Note that, since $p$ is an odd prime, $(a-1) s$ is odd if and only if $a$ and $k$ are both even. So (16) is real, and the fact that $\widehat{\mathbb{1}_{I}}(p-\gamma)=\widehat{\widehat{\mathbb{1}}_{I}(\gamma)}$ implies that the corresponding term for $p-\gamma$ is the same as for $\gamma$. This gives (15). A very similar calculation to (16) shows that

$$
F(I+t)=\sum_{\gamma=1}^{p-1} e^{-\pi i(2 t+a-1)(k-1) \gamma / p}\left|\widehat{\mathbb{1}_{I+t}}(\gamma)\right|^{k+1} \quad \text { for all } k \geqslant 3 .
$$

Given $r>0$ and $0 \leqslant \theta<2 \pi$, we write $\arg \left(r e^{\theta i}\right):=\theta$.

Proposition 11. Suppose that $p \geqslant 7$ is prime and $a \in[3, p-3]$. Then $\arg \left(\widehat{\mathbb{1}_{I^{\prime}}}(1)\right)$ is not an integer multiple of $\pi / p$.

Proof. Since $\widehat{\mathbb{1}_{A}}(\gamma)=-\widehat{\mathbb{1}_{\mathbb{Z}_{p} \backslash A}}(\gamma)$ for all $A \subseteq \mathbb{Z}_{p}$ and non-zero $\gamma \in \mathbb{Z}_{p}$, we may assume without loss of generality that $a \leqslant p-a$. Since $p$ is odd, we have $a \leqslant(p-1) / 2$.

Suppose first that $a$ is odd. Let $m:=(a-1) / 2$. Then $m \in\left[1, \frac{p-3}{4}\right]$. Observe that translating any $A \subseteq \mathbb{Z}_{p}$ changes the arguments of its Fourier coefficients by an integer multiple of $2 \pi / p$. So, for convenience of angle calculations, here we may redefine $I:=[-m, m]$ and $I^{\prime}:=\{-m-1\} \cup[-m+1, m]$. Also let $I^{-}:=[-m+1, m-1]$, which is non-empty. The argument of $\widehat{\mathbb{1}_{I^{-}}}(1)$ is 0 . Further, $\widehat{\mathbb{1}_{I^{\prime}}}(1)=\widehat{\mathbb{1}_{I^{-}}}(1)+\omega^{m+1}+\omega^{-m}$. Since $\omega^{m+1}, \omega^{-m}$ lie on the unit circle, the argument of $\omega^{m+1}+\omega^{-m}$ is either $\pi / p$ or $\pi+\pi / p$. But the bounds on $m$ imply that it has positive real part, $\operatorname{so} \arg \left(\omega^{m+1}+\omega^{-m}\right)=$ $\pi / p$. By looking at the non-degenerate parallelogram in the complex plane with vertices $0, \widehat{\mathbb{1}_{I^{-}}}(1), \omega^{m+1}+\omega^{-m}, \widehat{\mathbb{1}_{I^{\prime}}}(1)$, we see that the argument of $\widehat{\mathbb{1}_{I^{\prime}}}(1)$ lies strictly between that of $\widehat{\mathbb{1}_{I^{-}}}(1)$ and $\omega^{m+1}+\omega^{-m}$, i.e. strictly between 0 and $\pi / p$, giving the required.

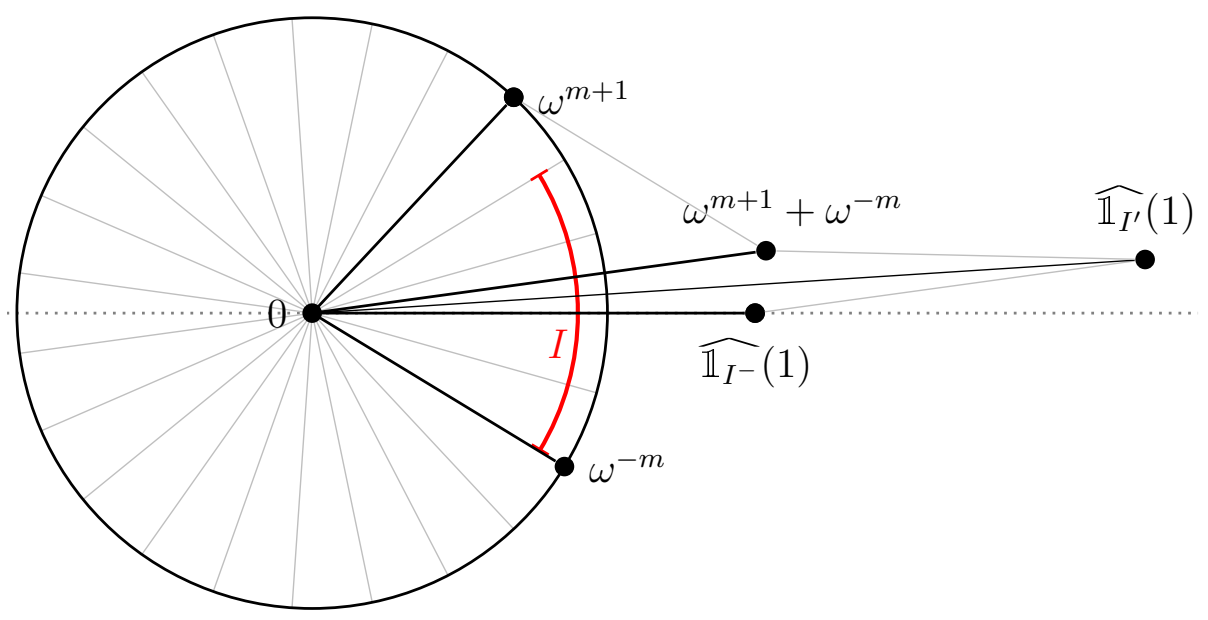

Suppose now that $a$ is even and let $m:=(a-2) / 2 \in\left[1, \frac{p-5}{4}\right]$. Again without loss of generality we may redefine $I:=[-m, m+1]$ and $I^{\prime}:=\{-m-1\} \cup[-m+1, m+1]$. Let also $I^{-}:=[-m+1, m]$, which is non-empty. The argument of $\mathbb{1}_{I^{-}}(1)$ is $-\pi / p$. Further, $\widehat{\mathbb{1}_{I^{\prime}}}(1)=\widehat{\mathbb{1}_{I^{-}}}(1)+\omega^{m+1}+\omega^{-(m+1)}$. The argument of $\omega^{m+1}+\omega^{-(m+1)}$ is 0 , so as before the argument of $\widehat{\mathbb{1}_{I^{\prime}}}(1)$ is strictly between $-\pi / p$ and 0 , as required. 
We say that an $a$-subset $A$ is a punctured interval if $A=I^{\prime}+t$ or $A=-I^{\prime}+t$ for some $t \in \mathbb{Z}_{p}$. That is, $A$ can be obtained from an interval of length $a+1$ by removing a penultimate point.

Lemma 12. Let $p \geqslant 7$ be prime and let $a \in\{3, \ldots, p-3\}$. Then the sets $I, I^{\prime} \subseteq \mathbb{Z}_{p}$ are not affine equivalent. Thus no punctured interval is affine equivalent to an interval.

Proof. Suppose on the contrary that there is $\alpha \in \mathcal{A}$ with $\alpha\left(I^{\prime}\right)=I$. Let a reflection mean an affine map $R_{c}$ with $c \in \mathbb{Z}_{p}$ that maps $x$ to $-x+c$. Clearly, $I=[a]$ is invariant under the reflection $R:=R_{a-1}$. Thus $I^{\prime}$ is invariant under the map $R^{\prime}:=\alpha^{-1} \circ R \circ \alpha$. As is easy to see, $R^{\prime}$ is also some reflection and thus preserves the cyclic distances in $\mathbb{Z}_{p}$. So $R^{\prime}$ has to fix $a$, the unique element of $I^{\prime}$ with both distance-1 neighbours lying outside of $I^{\prime}$. Furthermore, $R^{\prime}$ has to fix $a-2$, the unique element of $I^{\prime}$ at distance 2 from $a$. However, no reflection can fix two distinct elements of $\mathbb{Z}_{p}$, a contradiction.

We remark that the previous lemma can also be deduced from Proposition 11. Indeed, for any $A \subseteq \mathbb{Z}_{p}$, the multiset of Fourier coefficients of $A$ is the same as that of $x \cdot A$ for $x \in \mathbb{Z}_{p} \backslash\{0\}$, and translating a subset changes the argument of Fourier coefficients by an integer multiple of $2 \pi / p$. Thus for every subset which is affine equivalent to $I$, the argument of each of its Fourier coefficients is an integer multiple of $\pi / p$.

Let

$$
\rho(A):=\max _{\gamma \in \mathbb{Z}_{p} \backslash\{0\}}\left|\widehat{\mathbb{1}}_{A}(\gamma)\right| \text { and } \quad R(a):=\left\{\rho(A): A \in\left(\begin{array}{c}
\mathbb{Z}_{p} \\
a
\end{array}\right)\right\}=\left\{m_{1}(a)>m_{2}(a)>\ldots\right\} .
$$

Given $j \geqslant 1$, we say that $A$ attains $m_{j}(a)$, and specifically that $A$ attains $m_{j}(a)$ at $\gamma$ if $m_{j}(a)=\rho(A)=\left|\widehat{\mathbb{1}_{A}}(\gamma)\right|$. Notice that, since $\widehat{\mathbb{1}_{A}}(-\gamma)=\widehat{\widehat{\mathbb{1}_{A}}(\gamma)}$, the set $A$ attains $m_{j}(a)$ at $\gamma$ if and only if $A$ attains $m_{j}(a)$ at $-\gamma$ (and $\gamma,-\gamma \neq 0$ are distinct values).

As we show in the next lemma, the $a$-subsets which attain $m_{1}(a)$ are precisely the affine images of $I$ (i.e. arithmetic progressions), and the $a$-subsets which attain $m_{2}(a)$ are the affine images of the punctured interval $I^{\prime}$.

Lemma 13. Let $p \geqslant 7$ be prime and let $a \in[3, p-3]$. Then $|R(a)| \geqslant 2$ and

(i) $A \in\left(\begin{array}{c}\mathbb{Z}_{p} \\ a\end{array}\right)$ attains $m_{1}(a)$ if and only if $A$ is affine equivalent to $I$, and every interval attains $m_{1}(a)$ at 1 and -1 only;

(ii) $B \in\left(\begin{array}{c}\mathbb{Z}_{p} \\ a\end{array}\right)$ attains $m_{2}$ (a) if and only if $B$ is affine equivalent to $I^{\prime}$, and every punctured interval attains $m_{2}(a)$ at 1 and -1 only.

Proof. Given $D \in\left(\begin{array}{c}\mathbb{Z}_{p} \\ a\end{array}\right)$, we claim that there is some $D_{\text {pri }} \in\left(\begin{array}{c}\mathbb{Z}_{p} \\ a\end{array}\right)$ with the following properties:

- $D_{\text {pri }}$ is affine equivalent to $D$;

- $\rho(D)=\left|\widehat{\mathbb{1}_{D_{\text {pri }}}}(1)\right| ;$ and 
- $-\pi / p<\arg \left(\widehat{\mathbb{1}_{D_{\mathrm{pri}}}}(1)\right) \leqslant \pi / p$.

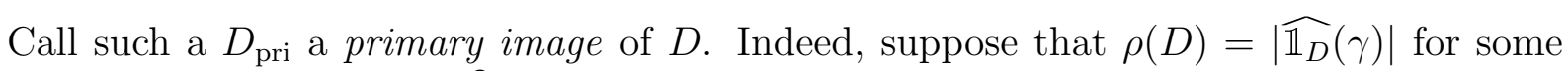
non-zero $\gamma \in \mathbb{Z}_{p}$, and let $\widehat{\mathbb{1}_{D}}(\gamma)=r^{\prime} e^{\theta^{\prime} i}$ for some $r^{\prime}>0$ and $0 \leqslant \theta^{\prime}<2 \pi$. (Note that we have $r^{\prime}>0$ since $p$ is prime.) Choose $\ell \in\{0, \ldots, p-1\}$ and $-\pi / p<\phi \leqslant \pi / p$ such that $\theta^{\prime}=2 \pi \ell / p+\phi$. Let $D_{\text {pri }}:=\gamma \cdot D+\ell$. Then

$$
\left|\widehat{\mathbb{1}_{D_{\mathrm{pri}}}}(1)\right|=\left|\sum_{x \in D} \omega^{-\gamma x-\ell}\right|=\left|\omega^{-\ell} \widehat{\mathbb{1}_{D}}(\gamma)\right|=\left|\widehat{\mathbb{1}_{D}}(\gamma)\right|=\rho(D),
$$

and

$$
\arg \left(\widehat{\mathbb{1}_{D_{\mathrm{pri}}}}(1)\right)=\arg \left(e^{\theta^{\prime} i} \omega^{-\ell}\right)=2 \pi \ell / p+\phi-2 \pi \ell / p=\phi,
$$

as required.

Let $D \subseteq \mathbb{Z}_{p}$ have size $a$ and write $\widehat{\mathbb{1}_{D}}(1)=r e^{\theta i}$. Assume by the above that $-\pi / p<$ $\theta \leqslant \pi / p$. For all $j \in \mathbb{Z}_{p}$, let

$$
h(j):=\Re\left(\omega^{-j} e^{-\theta i}\right)=\cos \left(\frac{2 \pi j}{p}+\theta\right),
$$

where $\Re(z)$ denotes the real part of $z \in \mathbb{C}$. Given any $a$-subset $E$ of $\mathbb{Z}_{p}$, we have

$$
H_{D}(E):=\sum_{j \in E} h(j)=\Re\left(e^{-\theta i} \sum_{j \in E} \omega^{-j}\right)=\Re\left(e^{-\theta i} \widehat{\mathbb{1}_{E}}(1)\right) \leqslant\left|\widehat{\mathbb{1}_{E}}(1)\right| .
$$

Then

$$
H_{D}(D)=\sum_{j \in D} h(j)=\Re\left(e^{-\theta i} \widehat{\mathbb{1}_{D}}(1)\right)=r=\left|\widehat{\mathbb{1}_{D}}(1)\right| .
$$

Note that $H_{D}(E)$ is the (signed) length of the orthogonal projection of $\widehat{\mathbb{1}_{E}}(1) \in \mathbb{C}$ on the 1-dimensional line $\left\{x e^{i \theta} \mid x \in \mathbb{R}\right\}$. As stated in (18) and (19), $H_{D}(E) \leqslant\left|\widehat{\mathbb{1}_{E}}(1)\right|$ and this is equality for $E=D$. (Both of these facts are geometrically obvious.) If $\left|\widehat{\mathbb{1}_{D}}(1)\right|=m_{1}(a)$ is maximum, then no $H_{D}(E)$ for an $a$-set $E$ can exceed $m_{1}(a)=H_{D}(D)$. Informally speaking, the main idea of the proof is that if we fix the direction $e^{i \theta}$, then the projection length is maximised if we take $a$ distinct elements $j \in \mathbb{Z}_{p}$ with the $a$ largest values of $h(j)$, that is, if we take some interval (with the runner-up being a punctured interval).

Let us provide a formal statement and proof of this now.

Claim 14. Let $\mathcal{I}_{a}$ be the set of length-a intervals in $\mathbb{Z}_{p}$.

(i) Let $M_{1}(D) \subseteq\left(\begin{array}{c}\mathbb{Z}_{p} \\ a\end{array}\right)$ consist of a-sets $E \subseteq \mathbb{Z}_{p}$ such that $H_{D}(E) \geqslant H_{D}(C)$ for all $C \in\left(\begin{array}{c}\mathbb{Z}_{p} \\ a\end{array}\right)$. Then $M_{1}(D) \subseteq \mathcal{I}_{a}$.

(ii) Let $M_{2}(D) \subseteq\left(\begin{array}{c}\mathbb{Z}_{p} \\ a\end{array}\right)$ be the set of $E \notin \mathcal{I}_{a}$ for which $H_{D}(E) \geqslant H_{D}(C)$ for all $C \in$ $\left(\begin{array}{c}\mathbb{Z}_{p} \\ a\end{array}\right) \backslash \mathcal{I}_{a}$. Then every $E \in M_{2}(A)$ is a punctured interval. 
Proof. Suppose that $0<\theta<\pi / p$. Then $h(0)>h(1)>h(-1)>h(2)>h(-2)>$ $\ldots>h\left(\frac{p-1}{2}\right)>h\left(-\frac{p-1}{2}\right)$. In other words, $h\left(j_{\ell}\right)>h\left(j_{k}\right)$ if and only if $\ell<k$, where $j_{m}:=(-1)^{m-1}\lceil m / 2\rceil$. Letting $J_{a-1}:=\left\{j_{0}, \ldots, j_{a-2}\right\}$, we see that

$H_{D}\left(J_{a-1} \cup\left\{j_{a-1}\right\}\right)>H_{D}\left(J_{a-1} \cup\left\{j_{a}\right\}\right)>H_{D}\left(J_{a-1} \cup\left\{j_{a+1}\right\}\right), H_{D}\left(J_{a-2} \cup\left\{j_{a-1}, j_{a}\right\}\right)>H_{D}(J)$

for all other $a$-subsets $J$. But $J_{a-1} \cup\left\{j_{a-1}\right\}$ and $J_{a-1} \cup\left\{j_{a}\right\}$ are both intervals, and $J_{a-1} \cup\left\{j_{a+1}\right\}$ and $J_{a-2} \cup\left\{j_{a-1}, j_{a}\right\}$ are both punctured intervals. So in this case $M_{1}(D):=$ $\left\{J_{a-1} \cup\left\{j_{a-1}\right\}\right\}$ and $M_{2}(D) \subseteq\left\{J_{a-1} \cup\left\{j_{a+1}\right\}, J_{a-2} \cup\left\{j_{a-1}, j_{a}\right\}\right\}$, as required.

The case when $-\pi / p<\theta<0$ is almost identical except now $j_{\ell}:=(-1)^{\ell}[\ell / 2]$ for all $0 \leqslant \ell \leqslant p-1$. If $\theta=0$ then $h(0)>h(1)=h(-1)>h(2)=h(-2)>\ldots>h\left(\frac{p-1}{2}\right)=$ $h\left(-\frac{p-1}{2}\right)$. If $\theta=-\pi / p$ then $h(0)=h(-1)>h(1)=h(-2)>\ldots=h\left(-\frac{p-1}{2}\right)>h\left(\frac{p-1}{2}\right)$.

We can now prove part (i) of the lemma. Suppose $A \in\left(\begin{array}{c}\mathbb{Z}_{p} \\ a\end{array}\right)$ attains $m_{1}(a)$ at $\gamma \in \mathbb{Z}_{p} \backslash\{0\}$. Then the primary image $D$ of $A$ satisfies $\left|\widehat{\mathbb{1}_{D}}(1)\right|=m_{1}(a)=\left|\widehat{\mathbb{1}_{A}}(\gamma)\right|$. So, for any $E \in$ $M_{1}(D)$,

$$
\left|\widehat{\mathbb{1}_{A}}(\gamma)\right|=\left|\widehat{\mathbb{1}_{D}}(1)\right| \stackrel{(19)}{=} H_{D}(D) \leqslant H_{D}(E) \stackrel{(18)}{\leqslant}\left|\widehat{\mathbb{1}_{E}}(1)\right|,
$$

with equality in the first inequality if and only if $D \in M_{1}(D)$. Thus, by Claim 14(i), $D$ is an interval, and so $A$ is affine equivalent to an interval, as required. Further, if $A$ is an interval then $D$ is an interval if and only if $\gamma= \pm 1$. This completes the proof of (i).

For (ii), note that $m_{2}(a)$ exists since by Lemma 12, there is a subset (namely $I^{\prime}$ ) which is not affine equivalent to $I$. By (i), it does not attain $m_{1}(a)$, so $\rho\left(I^{\prime}\right) \leqslant m_{2}(a)$. Suppose now that $B$ is an $a$-subset of $\mathbb{Z}_{p}$ which attains $m_{2}(a)$ at $\gamma \in \mathbb{Z}_{p} \backslash\{0\}$. Let $D$ be the primary image of $B$. Then $D$ is not an interval. This together with Claim 14(i) implies that $H_{D}(D)<H_{D}(E)$ for any $E \in M_{1}(D)$. Thus, for any $C \in M_{2}(D)$, we have

$$
m_{2}(a)=\left|\widehat{\mathbb{1}_{B}}(\gamma)\right|=\left|\widehat{\mathbb{1}_{D}}(1)\right|=H_{D}(D) \leqslant H_{D}(C) \leqslant\left|\widehat{\mathbb{1}_{C}}(1)\right| .
$$

with equality in the first inequality if and only if $D \in M_{2}(D)$. Since $C$ is a punctured interval, it is not affine equivalent to an interval. So the first part of the lemma implies that $\left|\widehat{\mathbb{1}_{C}}(1)\right| \leqslant m_{2}(a)$. Thus we have equality everywhere and so $D \in M_{2}(D)$. Therefore $B$ is the affine image of a punctured interval, as required. Further, if $B$ is a punctured interval, then $D$ is a punctured interval if and only if $\gamma= \pm 1$. This completes the proof of (ii).

We will now prove Theorem 3.

Proof of Theorem 3. Recall that $p \geqslant 7, a \in[3, p-3]$ and $k>k_{0}(a, p)$ is sufficiently large with $k \not \equiv 1(\bmod p)$. Let $I=[a]$. Given $t \in \mathbb{Z}_{p}$, write $\rho_{t}:=\left(\widehat{\mathbb{1}_{I+t}}(1)\right)^{k} \overline{\widehat{\mathbb{1}_{I+t}}(1)}$ as $r_{t} e^{\theta_{t} i}$, where $\theta_{t} \in[0,2 \pi)$ and $r_{t}>0$. Then (17) says that $\theta_{t}$ equals $-\pi(2 t+a-1)(k-1) / p$ modulo $2 \pi$. Increasing $t$ by 1 rotates $\rho_{t}$ by $-2 \pi(k-1) / p$. Using the fact that $k-1$ is invertible modulo $p$, we have the following. If $(a-1)(k-1)$ is even, then the set of $\theta_{t}$ for $t \in \mathbb{Z}_{p}$ is precisely $0,2 \pi / p, \ldots,(2 p-2) \pi / p$, so there is a unique $t$ (resp. a unique $t^{\prime}$ ) 
in $\mathbb{Z}_{p}$ for which $\theta_{t}=\pi+\pi / p$ (resp. $\left.\theta_{t^{\prime}}=\pi-\pi / p\right)$. Furthermore, $t^{\prime}=-(a-1)-t$ and $I+t^{\prime}=-(I+t)$; thus $I+t$ and $I+t^{\prime}$ have the same set of dilations. If $(a-1)(k-1)$ is odd, then the set of $\theta_{t}$ for $t \in \mathbb{Z}_{p}$ is precisely $\pi / p, 3 \pi / p, \ldots,(2 p-1) \pi / p$, so there is a unique $t \in \mathbb{Z}_{p}$ for which $\theta_{t}=\pi$. We call $t$ (and $t^{\prime}$, if it exists) optimal.

Let $t$ be optimal. To prove the theorem, we will show that $F(\xi \cdot(I+t))<F(A)$ (and so $\left.s_{k}(\xi \cdot(I+t))<s_{k}(A)\right)$ for any $a$-subset $A \subseteq \mathbb{Z}_{p}$ which is not a dilation of $I+t$.

We will first show that $F(I+t)<F(A)$ for any $a$-subset $A$ which is not affine equivalent to an interval. By Lemma 13(i), we have that $\left|\widehat{\mathbb{1}_{I+t}}( \pm 1)\right|=m_{1}(a)$ and $\rho(A) \leqslant m_{2}(a)$. Let $m_{2}^{\prime}(a)$ be the maximum of $\widehat{\mathbb{1}_{J}}(\gamma)$ over all length- $a$ intervals $J$ and $\gamma \in[2, p-2]$. Lemma 13(i) implies that $m_{2}^{\prime}(a)<m_{1}(a)$. Thus

$$
\left|F(I+t)-2\left(m_{1}(a)\right)^{k+1} \cos \left(\theta_{t}\right)-F(A)\right| \leqslant(p-1)\left(m_{2}(a)\right)^{k+1}+(p-3)\left(m_{2}^{\prime}(a)\right)^{k+1} .
$$

Now $\cos \left(\theta_{t}\right) \leqslant \cos (\pi-\pi / p)<-0.9$ since $p \geqslant 7$. This together with the fact that $k \geqslant k_{0}(a, p)$ and Lemma 13 imply that the absolute value of $2\left(m_{1}(a)\right)^{k+1} \cos \left(\theta_{t}\right)<0$ is greater than the right-hand size of $(20)$. Thus $F(I+t)<F(A)$, as required.

The remaining case is when $A=\zeta \cdot(I+v)$ for some non-optimal $v \in \mathbb{Z}_{p}$ and non-zero $\zeta \in \mathbb{Z}_{p}$. Since $s_{k}(A)=s_{k}(I+v)$, we may assume that $\zeta=1$. Note that $\cos \left(\theta_{t}\right) \leqslant$ $\cos (\pi-\pi / p)<\cos (\pi-2 \pi / p) \leqslant \cos \left(\theta_{v}\right)$. Thus

$$
\begin{aligned}
F(I+t)-F(I+v) & \leqslant 2\left(m_{1}(a)\right)^{k+1}\left(\cos \left(\theta_{t}\right)-\cos \left(\theta_{v}\right)\right)+(2 p-4)\left(m_{2}^{\prime}(a)\right)^{k+1} \\
& \leqslant 2\left(m_{1}(a)\right)^{k+1}(\cos (\pi-\pi / p)-\cos (\pi-2 \pi / p))+(2 p-4)\left(m_{2}^{\prime}(a)\right)^{k+1} \\
& <0
\end{aligned}
$$

where the last inequality uses the fact that $k$ is sufficiently large. Thus $F(I+t)<F(I+v)$, as required.

Finally, using similar techniques, we prove Theorem 5.

Proof of Theorem 5. Recall that $p \geqslant 7, a \in[3, p-3]$ and $k>k_{0}(a, p)$ is sufficiently large with $k \equiv 1(\bmod p)$. Let $I:=[a]$ and $I^{\prime}=[a-1] \cup\{a\}$.

Suppose first that $a$ and $k$ are both even. Let $A \subseteq \mathbb{Z}_{p}$ be an arbitrary $a$-set not affine equivalent to the interval $I$. By Lemma 13, $I$ attains $m_{1}(a)$ (exactly at $x= \pm 1$ ), while $\rho(A)<m_{1}(a)$. Also, $m_{2}^{\prime}(a)<m_{1}(a)$, where $m_{2}^{\prime}(a):=\max _{\gamma \in[2, p-2]}\left|\widehat{\mathbb{1}_{I}}(\gamma)\right|$. Thus

$$
\begin{aligned}
F(I)-F(A) & \stackrel{(14),(15)}{\leqslant} 2 \sum_{\gamma=1}^{\frac{p-1}{2}}(-1)^{\gamma}\left|\widehat{\mathbb{1}_{I}}(\gamma)\right|^{k+1}+\sum_{\gamma=1}^{p-1}\left|\widehat{\mathbb{1}_{A}}(\gamma)\right|^{k+1} \\
& \leqslant-2\left(m_{1}(a)\right)^{k+1}+(2 p-4)\left(\max \left\{m_{2}(a), m_{2}^{\prime}(a)\right\}\right)^{k+1}<0,
\end{aligned}
$$

where the last inequality uses the fact that $k$ is sufficiently large. So $s_{k}(a)=s_{k}(I)$. Using Lemma 13, the same argument shows that, for all $B \in\left(\begin{array}{c}\mathbb{Z}_{p} \\ a\end{array}\right)$, we have $s_{k}(B)=s_{k}(a)$ if and only if $B$ is an affine image of $I$. This completes the proof of Part 1 of the theorem. 
Suppose now that at least one of $a, k$ is odd. Let $A$ be an $a$-set not equivalent to $I$. Again by Lemma 13, we have

$$
\begin{aligned}
F(I)-F(A) & \geqslant \sum_{\gamma=1}^{p-1}\left|\widehat{\mathbb{1}_{I}}(\gamma)\right|^{k+1}-\sum_{\gamma=1}^{p-1}\left|\widehat{\mathbb{1}_{A}}(\gamma)\right|^{k+1} \\
& \geqslant 2\left(m_{1}(a)\right)^{k+1}-(p-1)\left(m_{2}(a)\right)^{k+1}>0 .
\end{aligned}
$$

So the interval $I$ and its affine images have in fact the largest number of additive $(k+1)$ tuples among all $a$-subsets of $\mathbb{Z}_{p}$. In particular, $s_{k}(a)<s_{k}(I)$.

Suppose that there is some $A \in\left(\begin{array}{c}\mathbb{Z}_{p} \\ a\end{array}\right)$ which is not affine equivalent to $I$ or $I^{\prime}$. (If there is no such $A$, then the unique extremal sets are affine images of $I^{\prime}$ for all $k>k_{0}(a, p)$, giving the required.) Write $\rho:=r e^{\theta i}=\widehat{\mathbb{1}_{I^{\prime}}}(1)$. Then by Lemma 13(ii), we have $r=m_{2}(a)$, and $\rho(A) \leqslant m_{3}(a)$. Given $k \geqslant 2$, let $s \in \mathbb{N}$ be such that $k=s p+1$. Then

$$
\left|F\left(I^{\prime}\right)-2 m_{2}(a)^{k+1} \cos (s p \theta)-F(A)\right| \leqslant(p-1) m_{3}(a)^{k+1}+(p-3)\left(m_{2}^{\prime}(a)\right)^{k+1} .
$$

Proposition 11 implies that there is an even integer $\ell \in \mathbb{N}$ for which $c:=p \theta-\ell \pi \in$ $(-\pi, \pi) \backslash\{0\}$. Let $\varepsilon:=\frac{1}{3} \min \{|c|, \pi-|c|\}>0$. Given an integer $t$, say that $s \in \mathbb{N}$ is $t$-good if $s c \in\left(\left(t-\frac{1}{2}\right) \pi+\varepsilon,\left(t+\frac{1}{2}\right) \pi-\varepsilon\right)$. This real interval has length $\pi-2 \varepsilon>|c|>0$, so must contain at least one integer multiple of $c$. In other words, for all $t \in \mathbb{Z} \backslash\{0\}$ with the same $\operatorname{sign}$ as $c$, there exists a $t$-good integer $s>0$. As $\operatorname{sp} \theta \equiv s c(\bmod 2 \pi)$, the sign of $\cos (s p \theta)$ is $(-1)^{t}$. Moreover, Lemma 13 implies that $m_{2}(a)>m_{3}(a), m_{2}^{\prime}(a)$. Thus, when $k=s p+1>k_{0}(a, p)$, the absolute value of $2 m_{2}(a)^{k+1} \cos (s p \theta)$ is greater than the right-hand side of (21). Thus, for large $|t|$, we have $F(A)>F\left(I^{\prime}\right)$ if $t$ is even and $F(A)<F\left(I^{\prime}\right)$ if $t$ is odd, implying the theorem by (14).

\section{Acknowledgements}

We are grateful to an anonymous referee for their careful reading of the manuscript and for many helpful comments.

\section{References}

[1] B. Bajnok, Additive combinatorics: A menu of research problems, CRC Press, Roca Baton, FL, 2018.

[2] P. Erdős, Extremal problems in number theory, Proc. Sympos. Pure Math., Vol. VIII, Amer. Math. Soc., Providence, R.I., 1965, pp. 181-189.

[3] B. Green and I. Z. Ruzsa, Sum-free sets in abelian groups, Israel J. Math. 147 (2005), $157-188$.

[4] S. Huczynska, G. L. Mullen, and J. L. Yucas, The extent to which subsets are additively closed, J. Combin. Theory Ser. A 116 (2009), 831-843. 
[5] E. Nazarewicz, M. O'Brien, M. O'Neill, and C. Staples, Equality in Pollard's theorem on set addition of congruence classes, Acta Arith. 127 (2007), 1-15.

[6] J. M. Pollard, Addition properties of residue classes, J. Lond. Math. Soc. 11 (1975), 147-152.

[7] W. Samotij and B. Sudakov, The number of additive triples in subsets of Abelian groups, Math. Proc. Camb. Phil. Soc. 160 (2016), 495-512.

[8] A. Terras, Fourier analysis on finite groups and applications, London Mathematical Society Student Texts, vol. 43, Cambridge University Press, Cambridge, 1999. 


\section{Corrigendum added March 122019}

After the publication of this paper, we learned that Theorem 1 follows from a result of Lev in [1] (Theorem 1) on solutions to the linear equation $x_{1}+\cdots+x_{k}=0$ in $\mathbb{Z}_{p}$.

\section{References}

[1] V. F. Lev, Linear equations over $\mathbb{Z} / p \mathbb{Z}$ and moments of exponential sums, Duke Math. J. 107 (2) (2001), 239-263. 